\title{
Functional Photoacoustic Microscopy
}

National Cancer Institute

\section{Source}

National Cancer Institute. Functional Photoacoustic Microscopy. NCI Thesaurus. Code C122709.

A hybrid imaging technique that combines the high-resolution advantage of ultrasonic detection of absorbed photons with the high-contrast advantage of optical imaging. It can provide better spatial resolution than pure optical imaging when the imaging depth is beyond $1 \mathrm{~mm}$. 